University of Nebraska - Lincoln

DigitalCommons@University of Nebraska - Lincoln

November 1989

\title{
THE PERIOD-DECLINE-RATE RELATION FOR PULSATING STARS
}

Edward G. Schmidt

University of Nebraska-Lincoln, eschmidt1@unl.edu

Kathryn $\mathrm{H}$. Wiese

University of Nebraska - Lincoln

Follow this and additional works at: https://digitalcommons.unl.edu/physicsschmidt

Part of the Physics Commons

Schmidt, Edward G. and Wiese, Kathryn H., "THE PERIOD-DECLINE-RATE RELATION FOR PULSATING STARS" (1989). Edward Schmidt Publications. 29.

https://digitalcommons.unl.edu/physicsschmidt/29

This Article is brought to you for free and open access by the Research Papers in Physics and Astronomy at DigitalCommons@University of Nebraska - Lincoln. It has been accepted for inclusion in Edward Schmidt Publications by an authorized administrator of DigitalCommons@University of Nebraska - Lincoln. 


\title{
THE PERIOD-DECLINE-RATE RELATION FOR PULSATING STARS
}

\author{
EDWARD G. SCHMIDT AND KaTHRYN H. WIESE \\ Behlen Observatory, Department of Physics and Astronomy, University of Nebraska, Lincoln, Nebraska 68588-0111 \\ Received 31 May 1989; revised 27 June 1989
}

\begin{abstract}
The relationships between the periods and the rate of decline in $V$ and $R$ for pulsating stars are investigated. It is shown that these relationships are useful for making preliminary estimates of periods for stars with little data. These estimates can then be used to optimize times of further observations.
\end{abstract}

\section{INTRODUCTION}

In order to use Cepheids in the determination of extragalactic distances, their periods must be known. This can be a serious limitation since the difficulties of observing these faint stars often restrict the amount of data available. Madore and Freedman (1985) suggested that the slope of the declining branch of the light curve or decline rate might be used to obtain approximate periods from limited amounts of data. Unfortunately, some photometry of Cepheids in NGC 6822 (Schmidt and Spear 1989) suggests the possibility of a different decline-rate-period relation and this would make the use of such a scheme risky. Nonetheless, the ease with which the decline rate can be determined from a small amount of data makes it a potentially useful parameter.

A random sampling of the stars listed in the General Catalogue of Variable Stars, 4th edition (Kholopov 1985, 1987, hereafter referred to as GCVS), shows that about $30 \%$ have no listed period. Seriously wrong periods are given for many others (see, for example, Loomis, Schmidt, and Simon 1988). In observing these stars, an erroneous period can lead the observer to obtain more frequent observations than necessary or to obtain them at intervals that are too long to yield a well-sampled light curve. Consequently, the efficiency of the observing program suffers. In such situations, the decline rate can be estimated from a few observations and an approximate period determined. This is then helpful in planning further observations. Additionally, when a large enough set of photometric data has been obtained, the decline rate can help to estimate the period range to be searched by some period-finding routine.

Sandage (1981) and Sandage, Katem, and Sandage (1981) have employed the rise time for RR Lyrae stars to characterize the shapes of the light curves. This is clearly closely related to the decline rate to the extent that there is a relatively restricted range in amplitude at any given period. Thus, departures from correlations between decline rate and period are useful as a means of identifying atypical light curves or distinguishing stars of various types.

The purpose of this paper is to extend the decline-rateperiod relation to a variety of commonly encountered types of variables for these purposes.

\section{THE PERIOD-DECLINE-RATE RELATION}

The decline rate $D$ (in magnitudes per day) was determined from published light curves. The sources of the photometry are listed in Table I. Although available light-curve data in $R$ is more limited than for $V$, the use of $R$ magnitudes is becoming more common due to the red sensitivity of modern detectors. Thus, we have included both $V$ and $R$ data in our analysis.

We have included Cepheids (both classical and type II), RR Lyrae stars, and Miras. These groups constitute $57 \%$ of the stars in the GCVS. The other large category of stars, eclipsing binaries, constitiutes about $29 \%$ of the catalog. However, they can generally be recognized from their characteristic light curves. Thus, our decline-rate-period relationship should be valid for the majority of the stars to which we will apply it.

$\log D(V)$ is plotted against the logarithm of the period in Fig. 1 while Fig. 2 is a plot of $\log D(R)$ against $\log P$. A good correlation exists for all the types of variables except the Miras and semiregular variables. The fact that these latter stars lie to the right of the others can be readily understood from their low temperatures; the $V$ and $R$ bands lie in the Wien tail of the blackbody curve and the surface fluxes are strongly dependent on the temperature. As is well known, this causes the $V$ amplitudes of Miras to be very large and will similarly increase their decline rates as seen here.

Aside from the Miras and semiregular variables, almost all of the stars fall between the two straight lines in the figures that are represented by

$$
\begin{aligned}
& \quad \log P=-0.92 \log D(V)+0.08 \pm 0.28 \\
& \text { for } V \text {, and } \\
& \quad \log P=-0.88 \log D(R)-0.04 \pm 0.24
\end{aligned}
$$

for $R$. Thus, with a measured decline rate in $V$ we can estimate the period within a range of \pm 0.28 in $\log P$ or within a factor slightly smaller than 2 if $\log D$ exceeds -1.0 so as to avoid the ambiguity introduced by the Miras. A slightly smaller uncertainty can be obtained using $R$ magnitudes.

TABLE I. Sources of photometric data.

\begin{tabular}{lcl}
\hline $\begin{array}{l}\text { Type of } \\
\text { variable }\end{array}$ & $\begin{array}{c}\text { Filter } \\
\text { band }\end{array}$ & \multicolumn{1}{c}{$\begin{array}{c}\text { Reference to } \\
\text { photometry }\end{array}$} \\
\hline Classical Cepheids & $V, R$ & Moffett and Barnes (1984) \\
Type II Cepheids & $V$ & Kwee and Diethelm (1984) \\
& $V, R$ & Loomis, Schmidt and Simon (1988) \\
RR Lyrae stars & $V$ & Lub (1977) \\
& $R$ & Liu and Janes (1989) \\
& $R$ & Loomis, Schmidt and Simon (1988) \\
Miras and semi- & $V, R$ & Cles S. (1982, 1986) \\
regular variables & & \\
\hline \hline
\end{tabular}




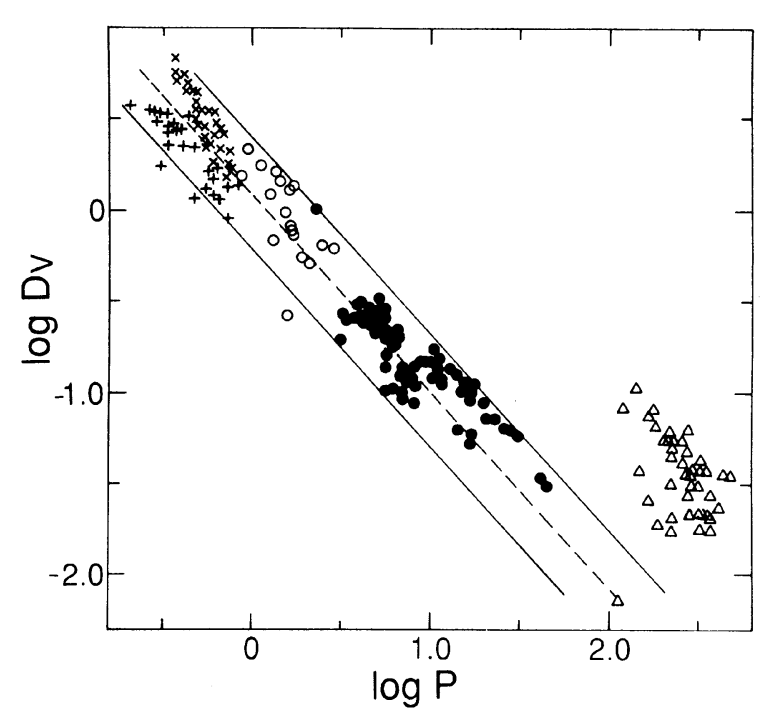

FIG. 1. Decline-rate-period plot for $V$. The symbols denote various types of variables as follows: filled circles, classical Cepheids; open circles, Type II Cepheids; $\times$, Bailey type ab RR Lyrae stars; + , Bailey type c RR Lyrae stars; triangles, Miras and semiregular variables. The solid lines are the boundaries of the correlation given by Eq. (1) while the dashed line is midway between the solid lines.

The width of the relationships given above are nearly twice that quoted by Madore and Freedman. However, an examination of Fig. 1 immediately reveals the cause of the discrepancy. Madore and Freedman only considered classical Cepheids with periods longer than 10 days. It can be seen that most such stars lie above the midline between the two solid lines in Fig. 1 and are thus confined to a region half the width of the full band. Furthermore, among the classical Cepheids only between periods of 5.5 and 8.0 days do the stars tend to reach the lower edge of the band. These stars lie

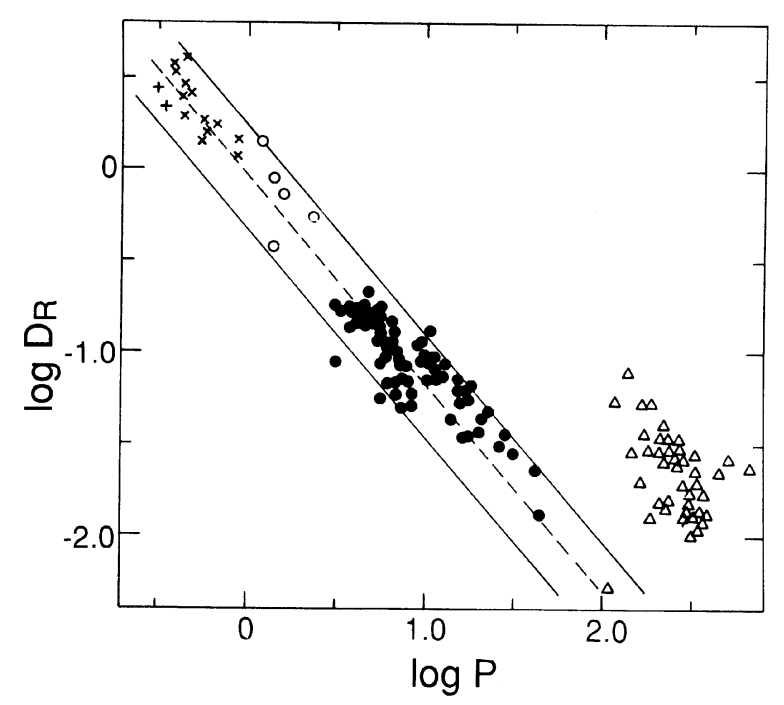

FIG. 2. Decline-rate-period plot for $R$. The symbols have the same meaning as in Fig. 1. between $\log D(V)=-0.8$ and $\log D(V)=-1.1$. It is well known that the light curves for Cepheids in this period range differ from those at longer and shorter periods in various respects (see, for example, Fernie and Chan 1986) and this behavior is attributed to mode resonances by Simon and Schmidt (1976). Thus, for stars with $\log D(V)$ less than 0 and outside of that narrow range, we can obtain periods accurate to \pm 0.15 in the log.

Among the RR Lyrae stars it can be seen that the dashed line separates most of the Bailey type c stars from the ab stars in Fig. 1. Thus, excluding the Bailey type $\mathrm{c}$ stars would also considerably narrow the band of stars in the RR Lyrae regime. However, a few light-curve points are not sufficient to recognize the type c stars and they are quite numerous. Thus, this is not a useful restriction and we must be satisfied with the accuracy implied by a width of \pm 0.28 in the log.

One star stands out in both Figs. 1 and 2 and deserves mention. The semiregular variable RR Car for which Cles (1982) quotes a period of 109 days has a much smaller decline rate than any other Mira or SRV in our sample at similar period. In fact, it is within an extension of the strip defined by the classical Cepheids in both diagrams. RR Car is classified as SRB in the GCVS which gives no period. The location of this star in the diagrams should be treated with some caution since the decline rate was determined from only two points 25 days apart and the periods of stars of this type are difficult to determine. However, since this star does seem to lie where a long-period Cepheid would be found, it might be worthwhile to undertake a study to verify its classification.

In applying the decline-rate-period relation to surveys of light curves, the first step is to observe a new star several times on the same night. From observations over perhaps 6 $\mathrm{hr}$ an initial estimate of the decline rate can be obtained from which a period can be estimated that is within a factor of 2 of the correct period provided the period is no longer than a few days. If this is the case, subsequent nights' observations can be planned using that period. If several observations are obtained on the following night, the period can then be refined sufficiently to allow the observations to be planned efficiently for that particular star. If the decline rate on the first night proves to be less than about 0.4 mag per day, it will be necessary to obtain further observations the following night. Since we now know that the period is longer than one day, observations spaced by a day will be adequate to determine the decline rate provided the period is less than about 10 days. If this is found to be the case, we are in a position to determine the preliminary period and proceed. If not, we need observations at a longer interval before we can do this.

It should be emphasized that the use of decline rates is solely for the purpose of obtaining preliminary period estimates which are reasonably correct most of the time. An accurate period must be determined later from the full set of data. It is realized that in some cases the initial period will be erroneous. This can happen if some of the points used in determining the slope of declining light do not lie on the declining branch of the light curve, if there are bumps or other features in the light curve which alter the slope locally, if we happen to be observing a star which falls outside of the envelopes in Figs. 1 or 2 or when photometric errors are too large or the time base too short to obtain an accurate decline rate. Nonetheless, the technique will work in a majority of cases and provides a useful tool for making observations in as efficient a manner as possible. 
Cles S., L. (1982). Astron. J. 87, 1791.

Cles S., L. (1986). Astrophys. J. Suppl. 60, 879.

Fernie, J. D., and Chan, S. J. (1986). Astrophys. J. 303, 766.

Kholopov, P. N. (1985). General Catalogue of Variable Stars, Fourth Edition, Vols. 1 and 2 (Nauka, Moscow).

Kholopov, P. N. (1987). General Catalogue of Variable Stars, Fourth Edition, Vol. 3 (Nauka, Moscow).

Kwee, K. K. and Diethelm, R. (1984). Astron. Astrophys. Suppl. 55, 77. Liu, T., and Janes, K. A. (1989). Astrophys. J. Suppl. 69, 593.

Loomis, C. L., Schmidt, E. G., and Simon, N. R. (1988). Mon. Not. R.
REFERENCES

Astron. Soc. 235, 1059

Lub, J. (1977). Astron. Astrophys. Suppl. 29, 345.

Madore, B. F., and Freedman, W. L. (1985). Astron. J. 90, 1104.

Moffett, T. J., and Barnes, T.G. (1984). Astrophys. J. Suppl. 55, 389.

Sandage, A. (1981). Astrophys. J. 248, 161.

Sandage, A., Katem, B., and Sandage, M. (1981). Astrophys. J. Suppl. 46, 41.

Schmidt, E. G., and Spear, G. G. (1989). Mon. Not. R. Astron. Soc. 236, 567.

Simon, N. R., and Schmidt, E. G. (1976). Astrophys. J. 205, 162. 